\section{DIN EN ISO/IEC 17025}

U. Zimmermann ${ }^{1}$ und A. Steinhorst ${ }^{2}$

${ }^{1}$ Gesundheit /Forensik, Deutsche Akkreditierungsstelle

GmbH (DAkkS), Frankfurt, Deutschland

${ }^{2}$ EA Secretariat, Paris, Frankreich

\section{Englischer Begriff ISO/IEC 17025}

Beschreibung Die Norm DIN EN ISO/IEC 17025 „Allgemeine Anforderungen an die Kompetenz von Prüf- und Kalibrierlaboratorien" wurde als Ergebnis der umfassenden Erfahrungen mit der Einführung von ISO/IEC Guide 25 und EN 45001 erarbeitet. Sie enthält alle Anforderungen, die Prüfund Kalibrierlaboratorien erfüllen müssen, wenn sie nachweisen wollen, dass sie ein Qualitätsmanagementsystem betreiben, technisch kompetent und fähig sind, fachlich fundierte Ergebnisse zu erzielen.
Akkreditierungsstellen, welche die Kompetenz von Prüfund Kalibrierlaboratorien anerkennen, verwenden diese Norm als Grundlage für ihre Akkreditierung. Inhaltlich enthält die Norm in 2 Abschnitten die Anforderungen an ein solides Managementsystem (Abschn. 4) und Anforderungen zum Nachweis der technischen Kompetenz für die Art von Prüfungen bzw. Kalibrierungen fest, die das Laboratorium durchführt (Abschn. 5).

Die speziellen Normen für medizinische Prüf- $(\triangleright$ DIN EN ISO 15189) und Kalibrierlaboratorien ( $\triangleright$ DIN EN ISO 15195) sind eng mit der Norm DIN EN ISO/IEC 17025 verbunden.

\section{Literatur}

DIN EN ISO/IEC 17025:2005,Allgemeine Anforderungen an die Kompetenz von Prüf- und Kalibrierlaboratorien“. Beuth-Verlag, Berlin 